\title{
A method to generate stable, collision free configurations for tensegrity based robots
}

\author{
Sergi Hernàndez Juan and Josep M. Mirats Tur
}

\begin{abstract}
Tensegrity structures appeared in the science community about half a century ago, but they have already been applied to several heterogeneous research fields, such as architecture, civil engineering, space and even biology. Such structures keep a stable volume in space due to an intricate balance of forces between a disjoint set of rigid elements (bars) and a continuous set of tensile elements (cables).

The use of tensegrity structures in robotics is still new and there exist only a handful of works about this subject. Some of their main features such as light weight, flexibility, energetic efficiency and redundancy, make them interesting candidates for both mobile robots and manipulators. In this paper, a new method to detect and avoid both internal collisions between the structure members and external collisions with the environment is presented. In this way, we are providing a fundamental tool to develop more complete form-finding procedures and pathplanning strategies for tensegrity structures.
\end{abstract}

\section{INTRODUCTION}

Robotics is a wide area of research in which very different kinds of robots have been proposed, from classical wheeled differential robots to modern parallel manipulators. Recently, a special class of pre-stressed structure called tensegrity has been introduced into the robotics domain ([1]) as a possible kind of new architecture in which to base a whole new type of robots.

The word tensegrity is an abbreviation for tensile integrity which was coined by Buckminister Fuller in the early 60's [2]. Tensegrities were created by people coming from the art community [3], being rapidly applied to other disciplines such as in the architectural context, for structures such as geodesic domes [4], or later in space engineering to develop deployable antennas [5] and masts [6].

Perhaps the most accepted definition for a tensegrity structure was given by Pugh [7]: A tensegrity system is established when a set of discontinuous compressive components interacts with a set of continuous tensile components to define a stable volume in space. The original definition only take into account two kind of elements: struts (compressive) and cables (tensile), with struts completely isolated from each other.

Afterwards, some authors have extended the original definition to include a third kind of element, the bar, which can withstand both tension and compression ([8]), and also to

$\mathrm{S}$. Hernàndez is a $\mathrm{PhD}$ student at the Institut de Robòtica i informàtica industrial (IRI), CSIC-UPC, C/Llorens i Artigas 4-6, 08028, Barcelona, spain. telf: +34 934015791 fax: +34934015750 shernandeiri.upc.edu

J. M. Mirats Tur is a full time researcher at the Institut de Robòtica i informàtica industrial (IRI), CSIC-UPC, C/Llorens i Artigas 4-6, 08028, Barcelona, spain. telf: +34 934054261 fax: +34934015750 jmiratseiri.upc.edu allow up to $k$ rigid bodies to be attached to a single node, which is known as class $k$ tensegrity ([9]).

From its origin, tensegrity structures have been mainly used for static applications where the length of all members is kept constant and actuation is only performed to compensate for external perturbations ([10], [11]). In the last decades, the tensegrity framework has been also used to build deployable structures ([6], [5]), although the tensegrity paradigm has not been fully exploited yet.

More recently, there has been an increasing interest on tensegrity structures from the robotics community which has lead to the first results in tensegrity based robots. [12] found the minimum time or minimum energy trajectory along a predefined path using several basic tensegrity structures put together to build a redundant manipulator.

[13], [1] developed locomotion algorithms for tensegrity based robots using genetic algorithms, and [14] proposed a self-propelled tensegrity worm which was actuated by the propagation of a longitudinal wave through the structure. However, only [1] actually built a working prototype.

Due to the increasingly complex tasks required for tensegrity structures, the need for a general motion planning algorithm arise. The first steps towards this goal were carried out by [15] and [16]. They defined the desired trajectory for the structures' center of mass ([15]) or for some of the structures' nodes ([16]) in the work space. Then, they divided the trajectory in a number of small segments, and for each of them, a stable configuration of the structure was found using optimization techniques.

Despite all these efforts, only [1] take collisions into account when simulating the behavior of each of the evolved locomotion algorithm in order to compute its fitness. The collision avoidance problem is important from two different points of view; first to avoid collision between elements of the structure (self-collision), and second, to be able to adapt the structure's shape to avoid contact with any external object.

We introduce in this paper a method to simultaneously detect and avoid both, self-collisions of the structure members, and collisions with environment obstacles (both modeled as simple geometrical shapes), while keeping the structure stable. The method is based on adding constraints to the form-finding optimization process which take into account all collision related issues.

This paper is organized as follows. First, the necessary theoretical concepts about tensegrity structures and standard path-planning techniques are presented in section II. Then, the proposed method to detect and avoid collisions while 
finding an stable solution for the structure is explained in detail in section III. In section IV two particular cases are presented: self-collision and obstacle collision avoidance. Finally, section V outlines some conclusions and future work.

\section{THEORETICAL BACKGROUND}

\section{A. TENSEGRITY STRUCTURES}

A tensegrity structure is fully parameterized by the spatial position of its nodes and by either the stresses or the rest lengths of its edges. In order for the structure to exist, these parameters have to be carefully chosen in order to comply with a restrictive set of constraints.

These constraints are given by the static equilibrium equations in all of its nodes (Eq. 1a), and the stress and geometrical compatibility of its edges (Eqs. 1b and 1c),

$$
\begin{array}{r}
\sum_{j \in A} k_{i j}\left(1-\frac{d_{i j 0}}{d_{i j}}\right)\left(\mathbf{p}_{i}-\mathbf{p}_{j}\right)=\mathbf{f}_{i}^{e x t}, A=\{n \in N \mid i j \in E\} \\
U_{i j} \leqslant d_{i j} \leqslant d_{i j 0} \leqslant L_{i j} i j \in C, \\
U_{i j} \leqslant d_{i j 0} \leqslant d_{i j} \leqslant L_{i j} i j \in S
\end{array}
$$

where $N$ is the set of all nodes, $E$ is the set of all edges (including the sets of cables $C$, bars $B$ and struts $S$ ), $U_{i j}$ and $L_{i j}$ are respectively the upper and lower distance bounds for each edge, $\mathbf{p}_{i}$ are the spatial coordinates for the $i-t h$ node and $d_{i j}$ and $d_{i j 0}$ are the real and rest length for the $i j$ edge respectively. Additional shape and symmetry constraints can be added as necessary. For a more detailed static review of tensegrity structures see [17].

Even for the most simple tensegrity structure in $\mathbb{R}^{3}$ (shown in Fig. 3), the dimension of the parameterization space, defined by the nodal positions and the edge rest lengths, is relatively high $(24$, if rigid movements are not taken into account). Additionally, due to the equality constraints presented in Eq. 1, most of the structure's parameters are tightly coupled, thus reducing the dimension of the solution space [15] (the space of feasible combinations of parameter values).

Due to the non-linear nature of the constraints in Eq. 1, it is not possible, in general, to find closed form expressions which take into account the couplings between the parameters of a tensegrity structure. Also, because of the reduced dimension of the solution space compared to the dimension of the parameterization space, the probability of randomly generating a feasible solution (a set of compatible nodal positions and feasible edge stresses) goes to 0. From the authors point of view, this issue is one of the most important open problems of tensegrity structures.

\section{B. PROBABILISTIC PATH-PLANNING}

The most common probabilistic path-planning approaches (PRM [18] and RRT [19]), are all based on sampling the configuration space for valid configurations.

The PRM approach first generates a great number of collision free samples inside the configuration space and then tries to link them by collision free paths. This process results in a roadmap of the collision free configuration space that can be used for fast multiple path-finding queries.
On the other hand the RRT approach grows a tree from the start or goal configuration (or both) until a collision free path is found. This method has to grow a new tree for each pair of start and goal configurations, and is more suited for single path-finding queries.

Both approaches depend on two main features: efficiently sampling the collision free configuration space, and efficiently finding a collision free path between feasible configurations (local planner). Both features are hard to accomplish for tensegrity structure because:

- All randomly generated configurations will not be initially feasible, so an optimization process is necessary for each of them. Furthermore, the resulting configuration might be discarded due to collisions.

- Due to the characteristics of the configuration space, finding feasible and collision free paths is difficult since the feasibility and collision constraints have to be checked for each point in the path.

So, developing a collision detection and avoidance method for tensegrity structures is the first step towards developing path-planning algorithms for tensegrity based robots.

\section{PROBLEM FORMULATION}

In order to solve the optimization problem, first the constrained problem is transformed into an unconstrained one (Eq. 2) by using quadratic and exponential penalty functions for the equality and inequality constraints respectively [20, sections 4.2.1 and 4.2.5].

$$
\min _{\mathbf{x}} f_{\text {cost }}(\mathbf{x})+c \sum_{i}\left\|h_{i}(\mathbf{x})\right\|^{2}+\sum_{i} \frac{1}{c}\left(e^{-c g_{i}(\mathbf{x})}-1\right),
$$

where $\mathbf{x}$ is the vector of optimization variables, $h_{i}(\mathbf{x})$ are the equality constraints, $g_{i}(\mathbf{x})$ are the inequality constraints and $f_{\text {cost }}(\mathbf{x})$ is the cost function in terms of the elastic energy of the structure.

The parameter $c$ in Eq. 2 is a positive penalty factor which weights the violation of the constraints and it is iteratively increased at each iteration. The possible values of this parameter range from 0.01 to 10000 , however the upper bound is never reached in any simulation.

Then a Quasi-Newton method is used to find the feasible solution to the unconstrained problem, which coincides with the solution to the original problem. In this case the inverse Hessian matrix is iteratively approximated by the BFGS method ([20, section 1.7]), and the Armijo rule is used to adaptively change the step size of the algorithm ([20, page 29]).

Additionally, it is possible to guarantee that the optimization algorithm always converges to a minimum of the function in Eq. 2, if it exists at all. This is achieved by some simple algebraic modifications to the problem constraints in Eq. 1 in order to eliminate some singular points as well as use some general results from non-linear programming.

Due to the relatively high computational cost associated to finding a single feasible configuration, it would be inefficient to ignore those configurations with collisions. Even so, without additional information, the optimization process will 
not be able to modify a potentially stable configuration to avoid collision.

It is necessary to include both the collision detection and avoidance inside the optimization procedure used to find stable configurations, so the solution configuration from the optimization process, if any, will be both stable and collision free.

To detect and avoid any possible collision, new constraints are added to the optimization problem. These new constraints, keep the minimum distance of two geometric objects greater or equal than 0 , or any given positive value (clearance between objects),

$$
D_{\text {min }}\left(\text { object }_{i}, \text { object }_{j}\right) \geqslant \epsilon, \forall \epsilon \geqslant 0 .
$$

During the optimization process, the value of the minimum distance is used as a monitor for detecting collisions, and its mathematical expression, in terms of the parameters of the structure, is used to move away from collision configurations.

At the moment only two kinds of collisions are taken into account:

- Collision between rigid members of the tensegrity structure (bars). Bars are modeled as infinite cylinders, parameterized as shown in Fig. 1 by the line equation $P(s)=P_{0}+s\left(P_{1}-P_{0}\right)=P_{0}+s u, \forall s$ and the radius $\left(r_{c}\right)$

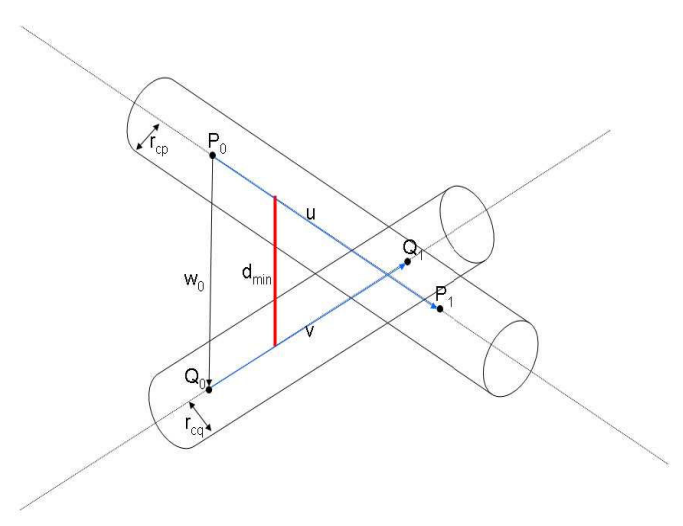

Fig. 1. Geometrical model for the bar-bar collision. Bars are modeled as infinite cylinders.

From Fig. 1, and using the fact that the minimum distance between two lines is defined by their common orthogonal vector, the minimum distance between two cylinders is

$$
D(P, Q)=\left|\mathbf{w}_{0}+\frac{(b e-c d) \mathbf{u}-(a e-b d) \mathbf{v}}{a c-b^{2}}\right| \geqslant r_{c p}+r_{c q},
$$

where $a=\mathbf{u u}^{T}, b=\mathbf{u} \mathbf{v}^{T}, c=\mathbf{v v}^{T}, d=\mathbf{u} \mathbf{w}_{0}^{T}$ and $e=\mathbf{v} \mathbf{w}_{0}^{T}$.

Eq. 4 is undefined when any two of the cylinders are parallel $\left(\frac{0}{\infty}\right)$, and the optimization method will not be able to handle this condition. To solve this problem, both sides of the inequality are multiplied by $\left(a c-b^{2}\right)^{2}$. Also, the 2-norm on the left handside of the inequality involves a square root which may introduce discontinuities in its gradient, so both sides are squared to guarantee the convergence of the optimization method [20]. After all the modifications, the bar-bar collision avoidance constraint is

$$
\begin{gathered}
\left|\mathbf{w}_{0}\left(a c-b^{2}\right)+(b e-c d) \mathbf{u}-(a e-b d) \mathbf{v}\right|^{2}- \\
-\left(a c-b^{2}\right)\left(r_{c p}+r_{c q}\right) \geqslant 0
\end{gathered}
$$

It is important to note that tensegrity bars, even if they are actuated, have a finite length, but Eq. 5 is only valid for infinite cylinders. However, for single staged tensegrity structures, such as the ones presented later in section IV, the artificial prolongation of the bars introduced by the constraints will not report any false bar-bar collisions, since the condition of minimum distance is always achieved inside the structure, in general stable configurations.

To avoid any possible collision between bars, it is necessary to check Eq. 5 for every pair of bars, so if a given tensegrity structure has $b$ bars, it is necessary to include $n_{\text {cil }}=\frac{b(b-1)}{2}$ constraints of this type to the optimization process.

- Collision between the nodes of the structure and obstacles. Environmental objects are modeled as infinite planes, parameterized by the normal vector $N^{p}=$ $\left(n_{x}^{P}, n_{y}^{P}, n_{z}^{P}\right)$ and its position in the space $O^{p}=$ $\left(o_{x}^{P}, n_{y}^{P}, n_{z}^{P}\right)$. The nodes of the tensegrity structure are modeled as spheres centered at $O^{s}=\left(o_{x}^{S}, o_{y}^{S}, o_{z}^{S}\right)$ with radius $r_{s}$, as shown in Fig. 2.

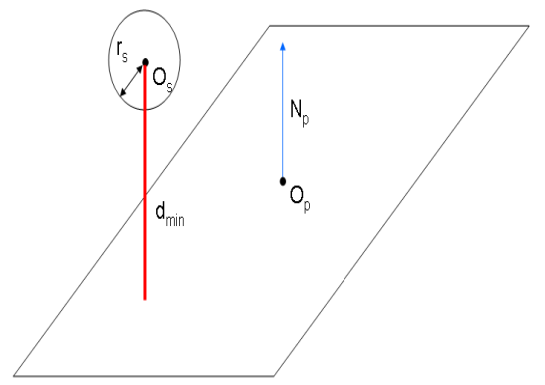

Fig. 2. Geometrical model for the node-obstacle collision. Obstacles are modeled as infinite planes and nodes as spheres.

In this case, the distance between a sphere and an infinite plane can be easily computed by evaluating the equation of the plane at the center of the sphere,

$n_{x}^{P}\left(o_{x}^{S}-o_{x}^{P}\right)+n_{y}^{P}\left(o_{y}^{S}-o_{y}^{P}\right)+n_{z}^{P}\left(o_{z}^{S}-o_{z}^{P}\right)-r_{s} \geqslant 0$.

By modeling the environment obstacles as infinite planes, it is enough to check collision with the nodes of the tensegrity structure. This is because they define the convex envelope of the structures used, and therefore, are the first part of the structure to contact any possible obstacle.

To avoid such collisions, it is necessary to check each node of the structure against each plane, so the total 
number of constraints that need to be included into the optimization process is $n_{\text {plane }}=n p$, where $n$ is the number of nodes and $p$ is the number of planes.

As introduced before in this section, both Eq. 5 and Eq. 6 must be included as inequality constraints to the optimization process presented before in Eq. 2.

\section{COLLISION AVOIDANCE}

The tensegrity structure shown in Fig. 3 has been used to validate the proposed method. It has its lower nodes fixed to ground, the 3 bars (thick blue lines) and the 3 vertical cables (red lines) are actuated, and the 3 upper nodes $(A, B$ and $C$ ) are free to move and are linked to each other by springs, with rest length fixed to $0.1 \mathrm{~m}$.

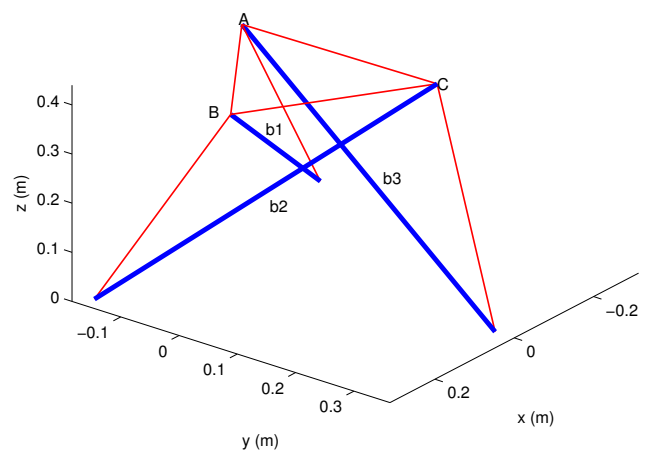

Fig. 3. Tensegrity structure used to validate the proposed method to avoid collisions.

In the next simulations, all actuated members (both bars and vertical cables) have a range of motion from $0.1 \mathrm{~m}$ to $2 \mathrm{~m}$, the diameter of the bars is $3.5 \mathrm{~cm}$, the diameter of the cables is negligible, the mass of the tensegrity structure is assumed to be located only at the nodes, and the Earth gravitational field is also considered. Also, the execution of the trajectory is assumed to be quasi-static, without taking into account any dynamics of the structure.

\section{A. OBSTACLE COLLISION AVOIDANCE}

The first case of study is the avoidance of collisions between environment obstacles and the structure. First, the structure holds its position with the center of mass of the upper triangle at $p_{\text {goal }}=(0,0,0.5)$ and an obstacle is approached, forcing the tensegrity structure to change its shape in order to stay at the desired position. Fig. 4 shows some configurations of the structure when an obstacle approaches.

In Fig. 4 it is possible to see how the tensegrity structure keeps the desired position (the center of mass of the upper triangle stays at $\left.p_{\text {goal }}=(0,0,0.5)\right)$, while its configuration is changed (compared to the default configuration shown in Fig. 3) in order to avoid the collision of the nodes with the plane.
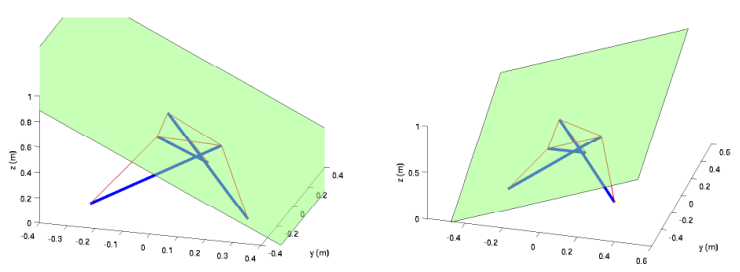

Fig. 4. Example of static tensegrity structure and mobile obstacles with collision avoidance

In this case a clearance of $1 \mathrm{~cm}$ has been used at each node to avoid direct contact between the nodes and the obstacle. Also, the diameter of the bars has been reduced to $1 \mathrm{~cm}$ to allow more range of motion to avoid the obstacle.

In a second simulation, the roles are reversed: the objects are static and the tensegrity structure is free to move through the work space. As shown in Fig. 5(a), two planes are placed on the work space as obstacles, and the structure tries to reach a position for the center of mass near them (marked as a red dot in Fig. 5(a)).

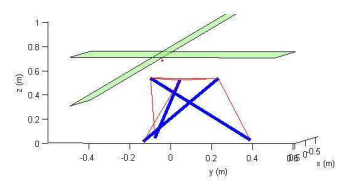

(a) Simulation setup with two planes as obstacles and the desired position (red dot).

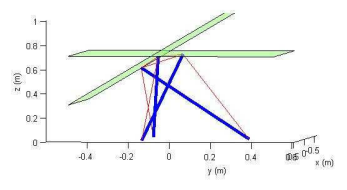

(b) Collision free and stable configuration at the desired position.
Fig. 5. Example of static obstacles and moving tensegrity structure with collision avoidance.

As shown in Fig. 5(b), the structure is capable of reaching the desired position for the center of mass while avoiding any collision with the obstacles. Note that the final configuration of the structure is quite different form the minimum energy configuration.

For probabilistic path-planning methods, this kind of obstacle collision avoidance is crucial to find random, feasible and collision free configurations of the tensegrity structure throughout the work space. Those configurations can then be connected together to build a roadmap. Then, by using the bar-bar collision avoidance presented later in section IV-B as a local planner, it would possible to find feasible, collision free trajectories between any two configurations of the roadmap.

Also, since the obstacle collision avoidance is integrated into the optimization process, it is possible to take advantage of the high level of flexibility this kind of structures have, thus maximizing the reachable space of the structure.

\section{B. SELF-COLLISION AVOIDANCE}

The second case of study is the avoidance of collisions between the elements of the structure themselves (self- 
collision). The simulation consists on obtaining the length variation of the actuated members required to follow a straight trajectory of the upper triangle's center of mass. The desired trajectory goes from the initial configuration at $p_{\text {start }}=(0,0,0.5)$ to a final position at $p_{\text {goal }}=(0,0.5,0.5)$ (plotted in green in both Fig. 6 and Fig. 7).

In order to compare the different configurations achieved by the structure when the self-collision of its members is considered, the proposed method is compared to the previous methods available on the literature ([15]). Fig. 6 shows the initial (dotted lines) and the final (solid lines) configurations of the tensegrity structure moving along the desired trajectory when collision avoidance is not taken into account. On the other hand, Fig. 7 shows the same information when the collision avoidance method proposed is used.

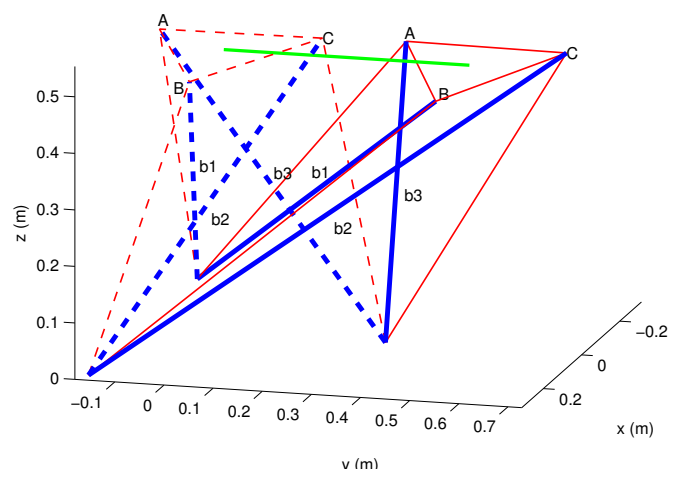

Fig. 6. Initial (dotted) and final configurations of the tensegrity structure moving along the desired trajectory (in green) without any self-collision avoidance.

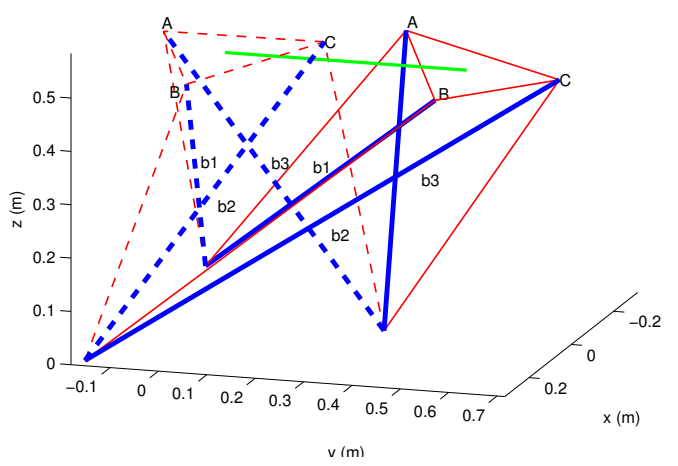

Fig. 7. Initial (dotted) and final configurations of the tensegrity structure moving along the desired trajectory (in green) using the proposed selfcollision avoidance method

In Fig. 7 the final configuration has changed compared to the one in Fig. 6 in order to avoid the collision of two bars ( $b 1$ and $b 2$ ), but the structure was still able to reach the desired final position for the center of mass. In the second case (using the collision avoidance method) it would be possible to use the obtained length variations of the actuated members to control a real prototype safely, while in the first case, the experiment may result in the destruction of the prototype due to excessive forces.

Fig. 8 shows the evolution of the relative distance between all three bars of the tensegrity structure with (in blue) and without (in red) the proposed collision avoidance method. In this figure it is easier to appreciate that the behavior of the structure is the same in both cases until the relative distance between bars $b 1$ and $b 2$ reaches $0.07 \mathrm{~m}$ (the two cylinders touch). From this point forward, the behavior of the structure is completely different.
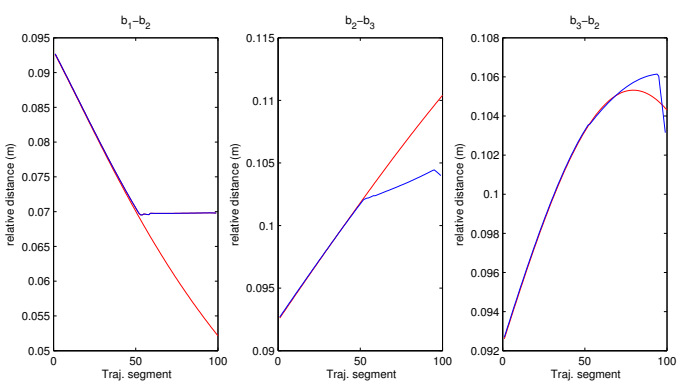

Fig. 8. Comparison of the relative distances between all three bars with (in blue) and without (in red) the proposed collision avoidance method.

It is important to note that in some cases, taking into account bar-bar collisions may prevent the structure to reach the goal configuration. Also, in the example shown in Fig. 7 , bars $b 1$ and $b 2$ actually touch each other but the relative distance keeps constant so preventing any damage to the structure. As introduced before, it is possible to avoid this by including a minimum clearance between any two bars.

The bar-bar collision avoidance is useful to plot a collision free trajectory between two feasible, collision free configurations. Therefore, it can be used as a local planner in one of the standard probabilistic methods presented in section II.

In the case that it would not be possible to go from one configuration to the other due to collisions of the edges of the structure themselves, it would be necessary to try to connect to nearby feasible and collision free configurations.

\section{CONCLUSIONS AND FUTURE WORK}

In this paper, a new method to deal with the collision avoidance, both between the tensegrity structure edges and between the structure and environment obstacles, has been presented. To our knowledge, it is the first attempt to actively avoid collision for tensegrity structures.

Simulations of the collision avoidance method applied to a simple tensegrity structure have also been presented which have allowed us to validate the proposed algorithm. It has been shown how this rather simple collision avoidance method for tensegrity structures can be used to find stable, collision free configurations in a given environment and also, as a local planner to find valid trajectories between any two of those configurations. 
The integration of the obstacle collision avoidance constraints into the optimization process also allows us to maximize the reachable space of a tensegrity based robot, because the configuration of the structure can be modified to fit narrow passages or overcome other obstacles impossible to avoid otherwise. However, bar-bar collision avoidance may invalidate some previously feasible configurations.

The method proposed in this paper models the obstacles as infinite planes and the bars of the structure as infinite cylinders. This has been enough to validate the proposed algorithm, but it will be necessary to take into account the finite length of all objects in the future. To do that it would be necessary to consider piecewise continuous functions, which increase the difficulty of the optimization process. In the future we also want to include the collision detection and avoidance of more complex objects in order to perform more complex tasks.

Currently, our group has a working prototype of a tensegrity structure (shown in Fig. 9) with only 3 actuators (the bars). This prototype is insufficient to test the proposed algorithm because it lacks enough controllable degrees of freedom. We are currently working on a new prototype which will have all edges actuated, both cables and bars, and will enable us to test the method in a real application.

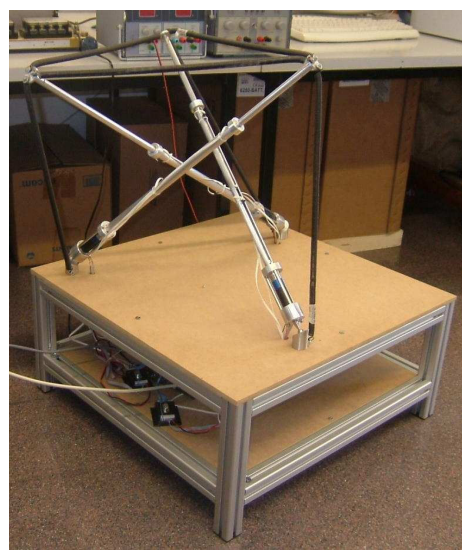

Fig. 9. Prototype developed by the tensegrity group at IRI.

Finally, if some kind of obstacle detection sensor can be integrated into the structure (such as ultrasonic or infrared rangers), it would be possible to actively change the shape of the tensegrity based robot in real time to avoid an approaching obstacle, because the time required to find the new configuration is under $0.2 \mathrm{~s}$, if a solution exists.

\section{ACKNOWLEDGMENTS}

This work has been partially funded by the Spanish Ministry of Education and Science under projects PROFIT CIT-020400-2007-78 and DPI2007-60858.

\section{REFERENCES}

[1] C. Paul, F. Valero-Cuevas, and H. Lipson, "Design and control of tensegrity robots for locomotion," IEEE Transactions on Robotics, vol. 22 , no. 5 , pp. $944-957$.
[2] R. Fuller, "Tensile-integrity structures," United States Patent 3063521, November 1962.

[3] K. Snelson, "Continuous tension, discontinuous compression structures," United States Patent 3169611, February 1965.

[4] R. Motro, "Tensegrity systems: the state of the art," Journal of Space Structures, vol. 7, no. 2, pp. 75-83, 1992.

[5] A. Tibert, "Deployable tensegrity structures for space applications," Ph.D. dissertation, Royal institute of technology, 2003.

[6] H. Furuya, "Concept of deployable tensegrity structures in space applications," Journal of Space Structures, vol. 7, no. 2, pp. 143-151, 1992.

[7] A. Pugh, An introduction to tensegrity. University of California Press, 1976.

[8] B. Roth and W. Whiteley, "Tensegrity frameworks," vol. 265, pp. 419446, 1981.

[9] R. Skelton, J. Helton, R.Adhikari, J. Pinaud, and W. Chan, "An introduction to the mechanics of tensegrity structures," in Proceedings of the 40th IEEE conference on Decision and control, 2001, pp. 42544258.

[10] W. Chan, D. Arbelaez, F. bossens, and R. Skelton, "Active vibration control of a three-stage tensegrity structure," in SPIE 11th Annual International Symposium on Smart Structures and Materials, San Diego, March 2004.

[11] M. Raja and S. Narayanan, "Active control of tensegrity structures under random excitation," Journal of Smart Materials and Structures, vol. 16, no. 3, pp. 809-817, 2007.

[12] J. Aldrich, "Control synthesis for a class of light and agile robotic tensegrity structures," Ph.D. dissertation, University of California, 2004.

[13] C. Paul, J. Roberts, H. Lipson, and F. Cuevas, "Gait production in a tensegrity based robot," in Proceedings of the International Conference on Advanced Robotics, 2005

[14] M. Masic and R. Skelton, "Open-loop control of class-2 tensegrity towers," in Proceedings of SPIE Modeling, Signal Processing, and Control, vol. 5383, 2004, pp. 298-308.

[15] J. Pinaud, M. Masic, and R. Skelton, "Path planning for the deployment of tensegrity structures," SPIE 10th Annual International Symposium on Smart Structures and Materials, 2003.

[16] J. van de Wijdeven and A. de Jager, "Shape change of tensegrity structures: design and control," in Proceedings of the American Control Conference, 2005.

[17] S. Hernàndez and J. Mirats-Tur, "Tensegrity frameworks: Static analysis review," Journal of Mechanism and Machine Theory, vol. 43, no. 7, pp. 859-881, 2008

[18] L. Kavraki, P. Svestka, J. Latombe, and M. Overmars, "Probabilistic roadmaps for path planning in high dimensional configuration spaces." IEEE Transactions on Robotics and Automation, vol. 12, 1996.

[19] S. LaValle, Rapidly-exploring Random Trees: A new tool for pathplanning. On-line, 1998.

[20] D. Bertsekas, Nonlinear programming. Athena Scientific, 1999. 